\title{
Anisotropy, in Density and in Distance, of the Dispersal of Yellow Rust of Wheat: Experiments in Large Field Plots and Estimation
}

\author{
S. Soubeyrand, J. Enjalbert, A. Sanchez, and I. Sache
}

First, second, third, and fourth authors: UMR207 Epidémiologie Végétale INRA-INA-PG, BP 01, 78850 Thiverval-Grignon, France; and first author: UR546 Biométrie, INRA, Domaine Saint-Paul Site AgroParc, 84914 Avignon Cedex 9, France. Accepted for publication 9 April 2007.

\section{ABSTRACT}

Soubeyrand, S., Enjalbert, J., Sanchez, A., and Sache, I. 2007. Anisotropy, in density and in distance, of the dispersal of yellow rust of wheat: Experiments in large field plots and estimation. Phytopathology 97:13151324.

Long-distance dispersal of spores generally presents anisotropy. This anisotropy can appear in the mean number of spores deposited along a given direction (anisotropy in density) and in the mean distance that a spore travels in a given direction (anisotropy in distance). Specific experiments together with a statistical methodology are proposed to study this effect. The experiments are based on the use of a point source of a traceable inoculum and susceptible trap plots in large resistant field plots.
The anisotropy is characterized by two functions: a directional density function and a mean distance function which are related with the anisotropies in density and distance, respectively. A nonparametric approach is developed to estimate these functions and to help in choosing a parametric model. Then, the parametric model is estimated. In two field experiments, migrations up to 175 and $225 \mathrm{~m}$ from the source were detected, with $\approx 25 \%$ of the trap plots infected. Whatever the experiment, the two estimated anisotropies presented different shapes (i.e., the number of spores dispersed in a given direction was not proportional to the mean distance travelled by these spores).

Additional keywords: Von Mises function.
The spatial heterogeneity of diseases spread by airborne spores has been addressed by several theoretical $(16,17,24,45)$ and experimental $(12,15,20,33,36-38,40)$ studies. However, relevant field data on larger scales of study are relatively sparse $(20,38)$. Yellow rust of wheat, caused by Puccinia striiformis and known to be a major cause of yield loss $(30,34)$, is one of the biological models which have been used in the experimental studies (12, $33,38)$. It is a typical focal disease, probably because of the steepness of the spore dispersal gradient (32). The spores which spread the pathogen are dispersed locally and over long distances. Most spores are liberated from sporulating lesions in clusters of 2 to 20 spores and are deposited closer to the source than they would be if dispersed individually (31-33). However, a few liberated spores are carried away by wind gusts to larger distances and form daughter (secondary) foci far away from the primary (mother) focus (45). For yellow rust, spore dispersal gradients from a point source have been assessed locally (up to 15 to $30 \mathrm{~m})(12,33)$ and, recently, on a larger scale (up to $80 \mathrm{~m}$ ) (38). Epidemiology and population genetics studies have revealed the continental dispersal of yellow rust (5).

Spore dispersal is anisotropic in windborne fungus (17). The resulting spatial pattern of disease spread from a primary disease focus is also anisotropic and mainly driven by prevailing winds, as shown in a field experiment with wheat yellow rust (12). The focus shape is the result of the distributions in direction and distance of the dispersal units (individual spores or spore clusters) (31). There is no indication that these two distributions should be identical. The classical approach for assessing the anisotropy of spore dispersal consists of sampling disease along lines radiating from the source $(12,38)$. Anisotropy in density cannot be correctly

Corresponding author: S. Soubeyrand

E-mail address: samuel.soubeyrand@avignon.inra.fr

doi:10.1094/PHYTO-97-10-1315

(C) 2007 The American Phytopathological Society assessed with such a design because the number of sampling directions is finite and the nonsampled area increases with increasing distance from the source $(6,16)$. A systematic, short-scale sampling of a big plot is no longer possible when the scale of the experiment increases. In contrast, anisotropy of spore dispersal both in density and distance can be assessed experimentally with a large array of small trap plots arranged around an artificially inoculated source plot $(20,37,43)$. The choice of the inoculum, the respective size of the source and trap plots, as well as the time schedule of the experiment must be decided according to the three following epidemiological features of a polycyclic, airborne disease like yellow rust: (i) dispersal on larger scales involves a very limited spore load and disease caused by these spores is often below practical detection thresholds; (ii) it is impossible to avoid contamination by external sources of inoculum (8), especially when the experimental scale increases; and (iii) the succession and juxtaposition of spore production cycles makes it difficult to differentiate contamination events caused by dispersal from an initial inoculum source or from secondary foci. Therefore, the experiment should be done with a significant trap plot area and an easily identifiable inoculum, and during a single generation of the pathogen.

The experiment proposed above allows one to visually assess anisotropy of spore dispersal. To summarise the data quantitatively, different kinds of mathematical models can be used. First, classical gradient functions (e.g., exponential and power law) can be fitted to data collected in several directions $(12,17)$; however, as noted above, anisotropy is not correctly addressed by these models. Second, mechanistic models based on the physical description of spore dispersal $(1,24)$ can be used to describe the transport of spores and can reflect anisotropy. However, the mechanistic models which have been proposed do not differentiate anisotropy in density and distance; on average, the spores travel further in their preferred dispersal direction. In addition, these models require a very detailed description of the physical environment (2), not easily obtainable in field conditions. Third, the so-called quasimechanistic models $(21,41,42)$ require a much 
simpler characterization of the environment, but they do not address the differential anisotropy in either density or distance.

In this article, we propose a combined experimental and statistical methodology of analysis of spore dispersal anisotropy in density and distance, as it occurs in the dispersal of wheat yellow rust. The experimental design is an array of trap plots in which spore dispersal from a source plot is assessed. In the modeling approach used to describe spore dispersal, anisotropy is defined by two functions characterizing dispersal in density and distance, respectively. The first function describes the direction taken by the spores during their travel, whereas the second one provides the expected distance between the spore's source and its deposition location, given the direction taken by the spore. The two anisotropy functions are not constrained by any prerequisite model; however, for assessing them, we propose a modeling and estimation framework. In a first step, a nonparametric analysis allowed us to determine the shape of the two anisotropy functions; in a second step, a parametric analysis was performed using the information gained from the first, nonparametric analysis.

\section{MATERIALS AND METHODS}

Field experiments. Field layout. The field experiments were performed during the 2001-02 cropping season in two locations of northwestern France, Le Neubourg (Upper Normandy; $49^{\circ} 15^{\prime} \mathrm{N}, 0^{\circ} 87^{\prime} \mathrm{W}$; elevation, $130 \mathrm{~m}$ above sea level), and Thiverval-Grignon (Isle of France; $48^{\circ} 50^{\prime} \mathrm{N}, 1^{\circ} 57^{\prime} \mathrm{E}$; elevation, $130 \mathrm{~m}$ above sea level), which are areas conducive to yellow rust epidemics. The distance between the two locations is $\approx 100 \mathrm{~km}$.

Wheat cv. Soissons, resistant to yellow rust, was sown in a 400by-75-m plot in Le Neubourg on 16 October 2001 and in a 250by-300-m plot in Thiverval-Grignon on 23 October 2001. Crop management followed the local standard practices. Two weeks after sowing, 1-by-1-m plots, hereafter referred to as the "trap plots," were delimited in the main plots on a $24-$ by-20-m grid in Le Neubourg and on a 25-by-24-m grid in Thiverval-Grignon (Figs. 1 and 2, respectively). There were 72 trap plots in Le Neubourg and 187 trap plots in Thiverval-Grignon. In Le Neubourg, the main plot was completely flat and the trap plots were arranged on a rectangular grid. In Thiverval-Grignon, the main plot was located on a hillside and the two axes of the grid made an angle of $63^{\circ}$. In each trap plot, the emerging seedlings of wheat cv. Soissons were removed with a hoe and four rows of wheat cv. Victo, highly susceptible to yellow rust, were sown manually. Emergence of wheat seedlings in the trap plots was homogeneous in Le Neubourg but heterogeneous in ThivervalGrignon. In Le Neubourg, the number of leaves per trap plot was assessed at 600. In Thiverval-Grignon, seedling density in the trap plots was visually assessed on 30 January 2002 on a 0-to-7 scale (no seedlings to fully established crop); the different levels correspond to different numbers of leaves ranking from 0 to 900 .
Using a resistant cultivar for the fields in which the trap plots of susceptible plants were included allowed us to reduce potential contamination from natural inoculum and prevented yield loss in those fields, which were harvested normally at the end of the crop season.

Inoculation procedure. A clonal isolate of $P$. striiformis, pathotype 106E139 (nomenclature according to Johnson et al.) (19), a pathotype no longer present in the natural airborne yellow rust populations and not virulent against the commercial wheat crops in use in the region $(10,44)$, was increased in the laboratory according to standard procedures (44). In brief, seedlings of wheat cv. Victo were grown in 5-by-5-cm pots until the unfolding of the second leaf and sprayed with $P$. striiformis spores suspended in mineral oil (Soltrol 130; Phillips Chemical Company, Phillips, TX). After $16 \mathrm{~h}$ in a dew chamber at $8^{\circ} \mathrm{C}$ in the dark, the pots with the inoculated seedlings were transferred in plastic batches $(40$ pots per batch, 20 batches) and kept in spore-proof cabinets inside a greenhouse. Two weeks after inoculation, rust sporulated profusely on the seedlings. The batches were sealed into polyethylene bags and brought into the plots for inoculation on 4 April 2002 in Le Neubourg and on 5 April 2002 in Thiverval-Grignon. On each site, 10 batches of sporulating seedlings constituting what will hereafter be called the "source plot" were set up in a 2.5-by-1.6-m area of the main plot equally sown with cv. Victo, in order to control the first symptom developments. In Le Neubourg, no prevailing wind directional effect was expected and the source plot was set up near the center of the main plot (Fig. 1). In Thiverval-Grignon, prevailing westerly winds were expected to contribute most to spore dispersal and the source plot was skewed westward from the center of the main plot (Fig. 2). The batches were taken out of the polyethylene bags, which were removed immediately from the field and discarded. Two weeks later, the batches of sporulating seedlings were placed into sealed polyethylene bags, removed from the plots, and discarded. In all cases, experimenters were careful to avoid any contact with the trap plots. In the trap plots and the surrounding field sown with the resistant cultivar, wheat was at the late tillering stage when the batches with sporulating seedlings were placed in the plots.

Disease assessment. All trap plots were checked for diseased leaves on 8 April in Le Neubourg and 10 April in ThivervalGrignon. All diseased leaves were counted, excised, and placed individually in a paper envelope. There was no attempt to count individual lesions on leaves because yellow rust does not form stripes on seedlings but, instead, groups of minute pustules covering the whole width of the leaf. The two fields were sprayed with a fungicide after the collection of the leaves.

Pathotype identification. Pathotypes were identified by the classic approach of determining the virulence or avirulence of the isolates on a set of differential hosts with known resistance genes or factors. The set of differential used for routine testing of the French isolates (10) is similar to the core set of differential culti-

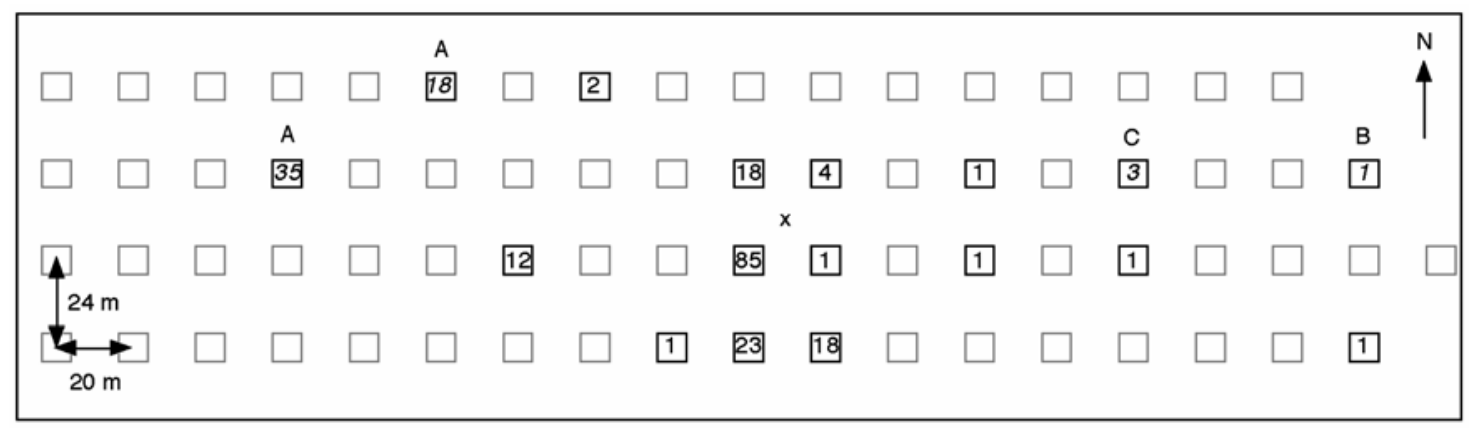

Fig. 1. Scale map of the field experiment performed in Le Neubourg. The location of the source plot is marked with X. Each square represents a 1-by-1-m trap plot. The size of the trap plots has been exaggerated for the sake of clarity. Numbers inside the squares are the numbers of diseased leaves collected, if any, at the end of the experiment. Numbers in italics indicate plots contaminated with exogenous inoculum. The letters stand for the identified pathotypes: A, 169E136-V17; B, 233E137-V17; and C, 237E141-V17. All other contaminated plots were found to be infected with pathotype 106E139 from the source plot. 
vars recommended by the European yellow rust experts (COST 817: 3 ). Due to the low genetic diversity of the populations of yellow rust in France $(10,13)$, it was assumed that the isolates harvested in the trap plots belonged either to (i) pathotype $106 \mathrm{E} 139$ used to inoculate the source plot, (ii) one of the few pathotypes usually found in the most recent surveys of the French populations of yellow rust (13), or (iii) pathotypes previously used in artificial inoculation in field experiments in Grignon. These pathotypes can be distinguished with a subset of 10 cultivars from the standard set of differential hosts (Table 1).

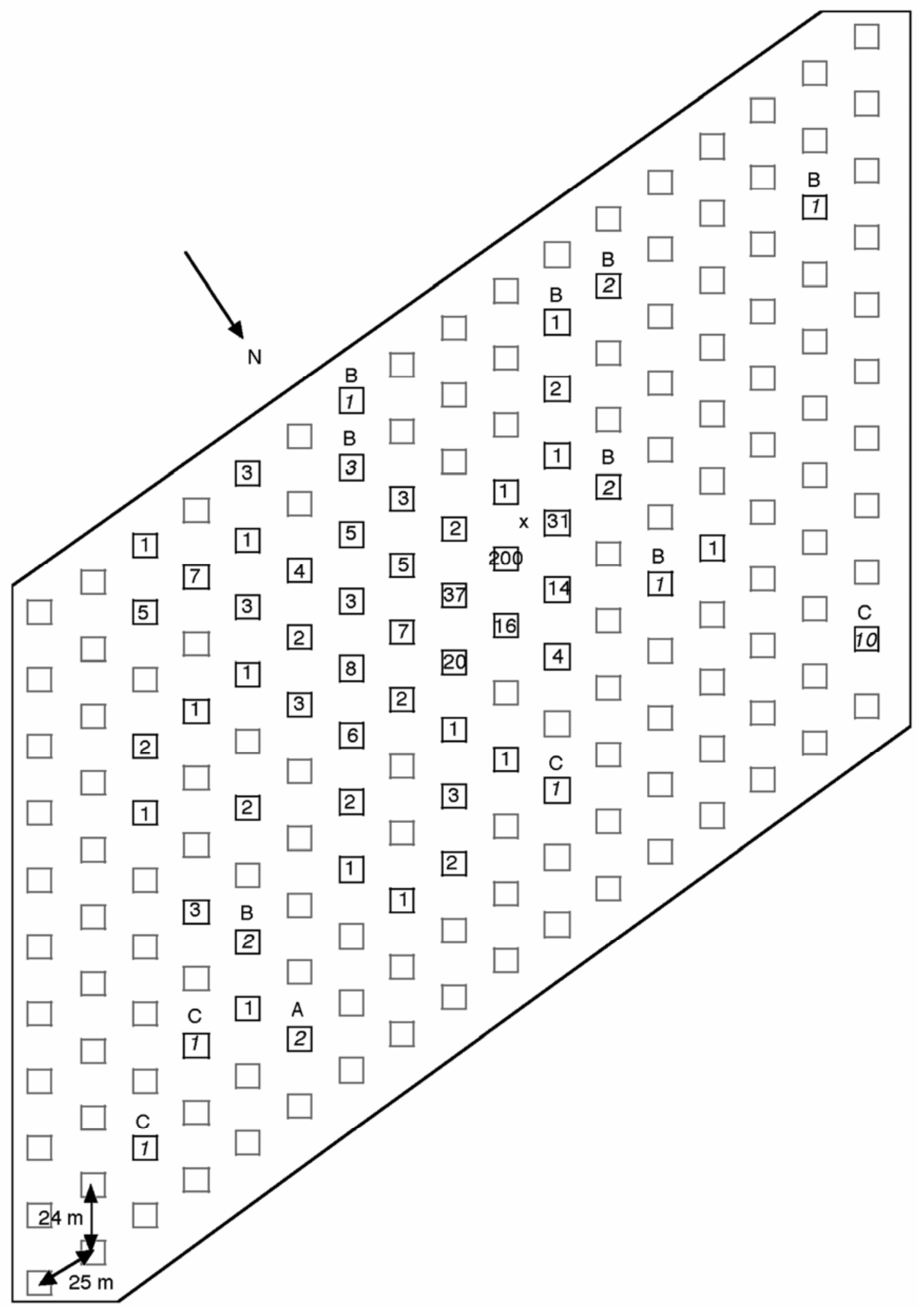

Fig. 2. Scale map of the field experiment performed in Thiverval-Grignon. The location of the source plot is marked with X. Each square represents a 1-by-1-m trap plot. The size of the trap plots has been exaggerated for the sake of clarity. Numbers inside the squares are the number of diseased leaves collected, if any, at the end of the experiment. Numbers in italics indicate plots contaminated with exogenous inoculum. The letters stand for the identified pathotypes: A, 45E140; B, 233E137; and C, 237E141-V17. All other contaminated plots were found to be infected with pathotype 106E139 from the source plot. 
Spores were increased by rubbing the leaf collected in the field against the primary leaf of a healthy seedling of wheat cv. Victo in order to produce the quantity of inoculum required for tests. The inoculated seedlings were handled as described above. Two weeks after inoculation, the newly formed spores were vacuumed and kept in a dessiccator filled with silica gel until use. Inoculation and incubation of the subset of cultivars with the spores to be tested followed the procedure described above. The inoculated seedlings then were placed into a growth chamber at $17^{\circ} \mathrm{C}$ for a 16-h light period with a light intensity of 300 mol quanta $\mathrm{m}^{-2} \mathrm{~s}^{-1}$ and at $14^{\circ} \mathrm{C}$ for an 8 -h dark period (44). Infection types on the differential hosts were assessed 15 days after inoculation using a 0-to-9 assessment scale (28). Compatible interactions (host susceptibility and pathogen virulence) were defined as infection types 6 to 7 (abundant sporulation, with chlorosis) and 8 to 9 (abundant sporulation without chlorosis).

Notations and definitions of the anisotropy functions. Notations used in the statistical analysis. Arbitrarily, the source plot center was assigned the coordinate $(0,0)$. Trap plots were indexed by $i$ in $\{1, \ldots, I\}$, where $I$ is the number of trap plots in the experimental field $(I=72$ for Le Neubourg and $I=187$ for ThivervalGrignon). In addition, $x_{1}, \ldots, x_{I}$ are the locations of the trap plot centers in Cartesian coordinates, $n_{1}, \ldots, n_{I}$ are the total numbers of leaves in the trap plots, and $v_{1}, \ldots, v_{I}$ are the numbers of leaves diseased by the inoculated pathotype 106E139 in the trap plots. Only leaves diseased by pathotype 106E139 were taken into account in the statistical analysis because we were interested in assessing the dispersal only from the source plot which released spores with pathotype 106E139.

For a spore dispersed from the source plot, let $(\Phi, R)$ denote, in polar coordinates, the random location of deposition of the spore. The angle $\Phi$ is called the random dispersal direction. The distance $R$ is called the random dispersal distance.

Uppercase symbols $Y_{i}$, $\Phi$, and $R$ were used to denote random variables whereas lowercase symbols $y_{i}, \varphi$, and $\rho$ were used to denote deterministic or observed values.

Directional density function. Let $f$ denote the directional density function. For direction $\varphi$ in $\left[0^{\circ}, 360^{\circ}[, f(\varphi) d \varphi\right.$ is the probability that the random dispersal direction $\Phi$ is in $[\varphi-d \varphi / 2, \varphi+d \varphi / 2]$, where $d \varphi$ is an infinitesimal arc length. In other words, it is the probability that the spore is deposited in an increasing width sector with angle $d \varphi$ radiating from the source in direction $\varphi$ (Fig. 3A). $f$ is the probability density function of the random direction $\Phi$ and reflects the frequency of directions taken by spores.

Mean distance function. Let the mean distance function describe, for any direction $\varphi$ in $\left[0^{\circ}, 360^{\circ}\right.$, how far from source a spore is deposited on average. Two versions, $g^{<}$and $g^{=}$, of this function are considered. First, $g^{<}(\varphi)$ is the mean dispersal distance of spores deposited over an increasing width sector centered at $\varphi$ (Fig. 3A). Second, $g^{=}(\varphi)$ is the mean dispersal distance of spores deposited over a constant width sector radiating from the source in direction $\varphi$ (Fig. 3A). When a disease gradient is estimated from disease measures observed on sampling units which

TABLE 1. Identification of the pathotypes collected in the experimental plots after inoculation of a subset of the standard set of differential hosts used to identify pathotypes of yellow rust in France

\begin{tabular}{|c|c|c|c|c|c|c|}
\hline \multirow[b]{2}{*}{ Differential host ${ }^{a}$} & \multirow[b]{2}{*}{ Gene $^{b}$} & \multicolumn{5}{|c|}{ Pathotype $^{c}$} \\
\hline & & $45 \mathrm{E} 140$ & $106 \mathrm{E} 139$ & 233E137-V17 & 233E169-V17 & 237E141-V17 \\
\hline Clement & Yr9, Yr2 & Avr & Avr & Vir & Vir & Vir \\
\hline Suwon92/Omar & Yr Suwon92/Omar & Avr & Vir & Vir & Vir & Vir \\
\hline Vilmorin 23 & Yr3 & Vir & Vir & Vir & Vir & Vir \\
\hline Heines Kolben & Yr6, Yr2 & Vir & Avr & Avr & Avr & Vir \\
\hline Lee & Yr7 & Avr & Vir & Avr & Avr & Avr \\
\hline Chinese 166 & Yr1 & Vir & Avr & Vir & Vir & Vir \\
\hline Carstens V & Yr Carstens V & Avr & Avr & Avr & Vir & Avr \\
\hline Compair & Yr8 & Avr & Avr & Avr & Avr & Avr \\
\hline Hybrid 46 & Yr4 & Avr & Vir & Vir & Vir & Vir \\
\hline VPM 1 & Yr17 & Avr & Avr & Vir & Vir & Vir \\
\hline
\end{tabular}

a See the complete standard set $(3,28)$.

b Nomenclature of the genes for resistance to yellow rust according to McIntosh et al. (26).

c Pathotype designation according to Johnson et al. (19); -V17: virulent for Yr17. Vir = virulent and Avr = avirulent on a 0-to-9 scale (18).
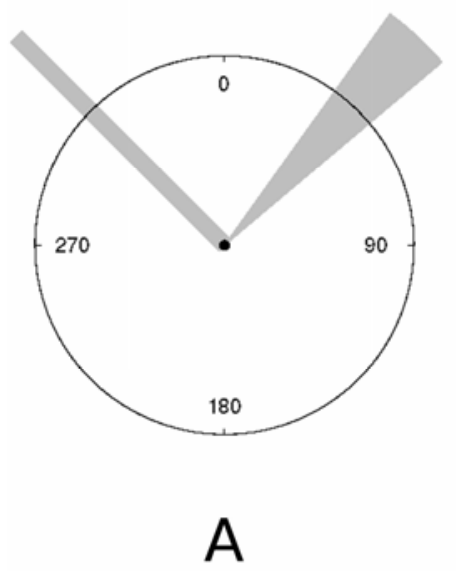

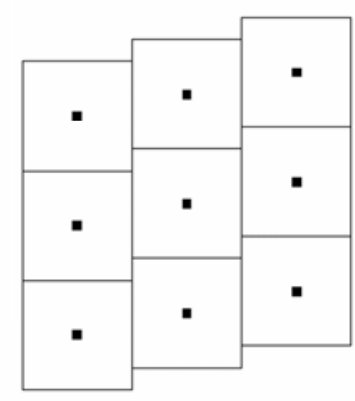

B

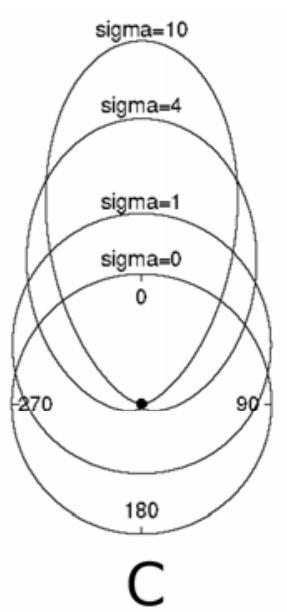

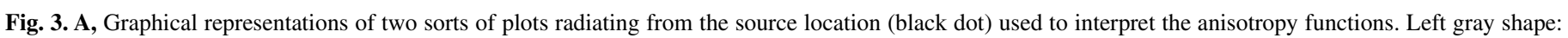

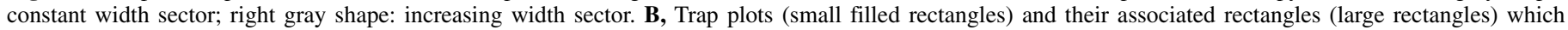

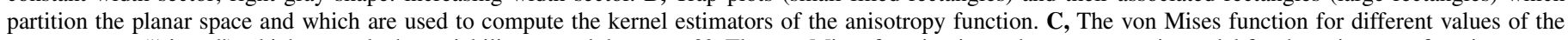
parameter $\sigma$ ("sigma") which controls the variability around the mean $0^{\circ}$. The von Mises function is used as a parametric model for the anisotropy functions. 
are lined up in a direction $\varphi$ and have similar shapes and sizes (38), then the version of the mean dispersal distance which can be estimated is $g=(\varphi)$.

Nonparametric framework. Kernel estimator for the directional density function $f$. A kernel density estimator, $\hat{f}$, was built to estimate the directional density function $f$. A kernel density estimator can be thought of as a smoothed version of an histogram and is obtained by computing kinds of moving averages. Let $\Phi_{1}, \ldots, \Phi_{N}$ be random dispersal directions of $N$ spores. Assume $\Phi_{1}, \ldots, \Phi_{N}$ are independent and identically drawn from the probability distribution $f$. The kernel density estimator of $f(\varphi)$, $\varphi \in\left[0^{\circ}, 360^{\circ}[\right.$, is defined by

$$
\hat{f}(\phi)=\frac{1}{N} \sum_{n=1}^{N} \frac{1}{b} K\left(\frac{\varphi-\Phi_{n}}{b}\right)
$$

where $K$ is called the kernel function and $b$ is the bandwidth $(4,14,39)$. Each dispersal direction $\Phi_{n}$ contributes to the value of $\hat{f}(\varphi)$ at a level depending on the closeness between $\varphi$ and $\Phi_{n}$ : the closer $\Phi_{n}$ from $\varphi$, the larger its contribution. Therefore, if there are a lot of $\Phi_{n}$ close to $\varphi$, then the value of the estimated density $\hat{f}$ at $\varphi$ is high. On the contrary, if there are few $\Phi_{n}$ close to $\varphi$ then $\hat{f}(\varphi)$ is low. The closeness is defined through the kernel function $K$ and the bandwidth $b$.

Kernel estimator for the mean distance function $g^{<}$. A kernel smoother, $\hat{g}^{<}$, was used to estimate the mean distance function $g^{<}$. Let $\Phi_{1}, \ldots, \Phi_{N}$ and $R_{1}, \ldots, R_{N}$ be the random dispersal directions and distances, respectively, of $N$ spores. Assume pairs $\left(\Phi_{1}, R_{1}\right) \ldots,\left(\Phi_{N}, R_{N}\right)$ are independent and identically distributed. The kernel smoother of $g^{<}(\varphi), \varphi \in\left[0^{\circ}, 360^{\circ}\right.$, is

$$
\hat{g}^{<}(\phi)=\sum_{n=1}^{N} \frac{K\left(\frac{\varphi-\Phi_{n}}{b}\right)}{\sum_{m=1}^{N} K\left(\frac{\varphi-\Phi_{m}}{b}\right)} R_{n}
$$

where $K$ is the kernel function and $b$ is the bandwidth $(4,18)$. The estimator $\hat{g}^{<}(\varphi)$ of the expected dispersal distance is a weighted mean of dispersal distances $R_{1}, \ldots, R_{N}$ : the closer $\Phi_{n}$ from $\varphi$, the larger the weight of $R_{n}$ in the mean.

Choices of the kernel function and the bandwidth. The kernel function $K$ usually is taken to be positive, symmetric, and $K(u)$ usually decreases as $|u|$ increases. Thus, the contribution of observation $n$ in the calculation of $\hat{f}(\varphi)$ or $\hat{g}^{<}(\varphi)$ decreases as the difference between $\Phi_{n}$ and $\varphi$ increases. For both the kernel density estimator $\hat{f}$ and the kernel smoother $\hat{g}^{<}$, the kernel function

$$
K(u)= \begin{cases}0.9375\left(1-u^{2}\right)^{2} & \text { if }-1 \leq u \leq 1 \\ 0 & \text { otherwise }\end{cases}
$$

suggested by Fisher (14) was used. Moreover, the bandwidth $b$ was selected by leave-one-out cross-validation $(18,23)$. Leaveone-out cross-validation involves using $N-1$ observations for computing the estimator, and using the remaining observation for validation; this is repeated in turn as each observation is used once as validation data.

Computation of the kernel estimators. Ideally, the kernel density estimator and the kernel smoother should be computed by sampling, randomly, $N$ spores and by observing their deposition locations $\left(\Phi_{1}, R_{1}\right), \ldots,\left(\Phi_{N}, R_{N}\right)$. However, data at our disposal are numbers of diseased leaves in trap plots. Therefore, the estimators of the anisotropy functions were computed as follows. The experimental field was partitioned into rectangles $\mathfrak{R}_{1}, \ldots, \mathfrak{R}_{I}$ centered on the trap plots (Fig. 3B). For each trap plot $i$, the observed number of diseased leaves $y_{i}$ was assumed to be proportional to the number of spores deposited in $\mathfrak{R}_{i}$. For each index $i, y_{i}$ locations were randomly generated independently and uniformly in $\mathfrak{R}_{i}$. $N=\sum_{i=1}^{I} y_{i}$ locations, say $\left(\Phi_{1}, R_{1}\right), \ldots,\left(\Phi_{N}, R_{N}\right)$, were obtained, and estimators $\hat{f}$ and $\hat{g}^{<}$(equations 1 and 2) were computed using these locations. With this procedure, $\hat{f}$ and $\hat{g}^{<}$depend on the randomly generated locations; therefore, the procedure was repeated 100 times, producing 100 estimates for $f$ and $g^{<}$, and the final estimates for these functions were the means of the 100 estimates.

Parametric framework. A parametric model was proposed to estimate both the directional density function $f$ and the mean distance function $g$. First, an underlying model integrating $f$ and $g^{=}$was defined to model numbers of lesions on leaves. Then, the underlying model was derived to get a model adapted to our data which consists of numbers of diseased leaves in trap plots.

Underlying model. An underlying model describes the number of lesions per leaf. For any leaf located at $x$, the number of lesions is assumed to follow a Poisson distribution with mean $S(x)$, where $S(x)$ is called infectious potential at $x . S(x)$ measures the expected quantity of spores which are deposited at location $x$ and, potentially, can infect a leaf.

The infectious potential at location $x$, generated by a point source located at $(0,0)$, integrates the anisotropy functions $f$ and $g^{=}$:

$$
S(x)=s_{0} \frac{f(\varphi)}{g^{=}(\varphi)^{2}} \exp \left\{-\frac{\rho}{g^{=}(\varphi)}\right\}
$$

where $(\varphi, \rho)$ are the polar coordinates of $x$. In equation 3 , parameter $s_{0}$ represents the strength of the source of spores. The quantity $S(x) d x / s_{0}$ is the probability that a spore dispersed by the source is deposited in the infinitesimal rectangular area $d x$ centered at $x$. The infectious potential function is the two-dimensional version of the exponential dispersal gradient $(1,38)$. Note that, in this parameterization, it can be shown that $\hat{g}^{<}(\varphi)=2 g^{=}(\varphi)$ for any $\varphi$ in $\left[0^{\circ}, 360^{\circ}[\right.$.

Parametric forms for the anisotropy functions. The nonparametric estimation of the anisotropy functions suggested that unimodal parametric forms could be used. The directional density function $f$ was assumed to be a von Mises function parameterized by $\mu$ and $\sigma$ (14)

$$
\begin{aligned}
& f(\phi)=\left\{2 \pi I_{0}(\sigma)\right\}^{-1} \exp \{\sigma \cos (\varphi-\mu)\} \\
& I_{0}(\sigma)=(2 \pi)^{-1} \int_{0}^{2 \pi} \exp \{\sigma \cos (\theta-\mu)\} d \theta
\end{aligned}
$$

This is a unimodal density distribution over $\left[0^{\circ}, 360^{\circ}[. \mu\right.$ is the expected dispersal direction and $\sigma$ controls the variability of dispersal directions around $\mu . \sigma=0$ means that dispersal directions are uniformly distributed in $\left[0^{\circ}, 360^{\circ}\right.$, and $\sigma=\infty$ means that dispersal directions are all equal to $\mu$. The von Mises function for different values of $\sigma$ is shown in Figure 3C. The von Mises function is a unimodal function (i.e., there is only one main dispersal direction). It is considered as the analog on the circle, or on the segment $\left[0^{\circ}, 360^{\circ}\right.$, of the Gaussian distribution on the real line; it is the most common model for unimodal samples of circular data (14).

The distance dispersal function $g^{=}$was assumed to be proportional to a von Mises function parameterized by $\nu$ and $\kappa$

$$
g(\varphi)=g_{0}\left\{2 \pi I_{0}(\kappa)\right\}^{-1} \exp \{\kappa \cos (\varphi-\nu)\}
$$

$v$ is the direction at which the mean dispersal distance is the larger, that is $g_{0} \exp (\kappa)\left\{2 \pi I_{0}(\kappa)\right\}^{-1}$. $\kappa$ controls the variability of the expected dispersal distance as the dispersal direction varies. $\kappa=0$ means that dispersal distances are the same whatever the dispersal directions. $g_{0}$ is a multiplicative constant measuring how far from source spores are deposited.

Derivation of a model adapted to the data. An underlying model for the numbers of lesions on leaves was defined in the 
previous paragraphs. A model, adapted to our data, was derived from the underlying model. Suppose the source plot is small enough to be viewed as a point source. Then, it generates an infectious potential with the form of equation 3. Consider trap plot $i$ centred at $x_{i}$. Suppose the trap plot is small enough to consider that the infectious potential $S$ is constant over the trap plot and equals $S\left(x_{i}\right)$. Because the number of lesions on a leaf at $x_{i}$ is Poisson distributed with mean $S\left(x_{i}\right)$, the number of diseased leaves $Y_{i}$ in trap plot $i$ follows a binomial distribution with size $n_{i}$ and probability $p\left(x_{i}\right)$

$$
Y_{i} \sim \text { Binomial }\left\{n_{i}, p\left(x_{i}\right)\right\}
$$

where

$$
p\left(x_{i}\right)=1-\exp \left\{-S\left(x_{i}\right)\right\}
$$

Recall that $n_{i}$ is the total number of leaves located in trap plot $i$. $p\left(x_{i}\right)$ equals $1-\exp \left\{-S\left(x_{i}\right)\right\}$ because it is the probability for a leaf at $x_{i}$ to be diseased or, in other words, the probability that at least one lesion is on the leaf. Note that equation 6 is a familiar incidence-density relationship (29).

Estimation, tests, and confidence intervals for the parameters. In the model, there are six parameters: the source strength $s_{0}$, the direction parameters $\mu$ and $\sigma$, and the distance parameters $v$, $\kappa$, and $g_{0}$. Let the vector of parameters be $\lambda=\left(s_{0}, \mu, \sigma, \nu, \kappa, g_{0}\right)$. $\lambda$ is estimated by maximum of likelihood (35). Intuitively, the maximum likelihood estimator, $\hat{\lambda}=\left(\hat{s}_{0}, \hat{\mu}, \hat{\sigma}, \hat{v}, \hat{\kappa}, \hat{g}_{0}\right)$, of $\lambda$ is the set of parameters which has the most support from the data in the sense of being the most likely set of parameters, given the data. Formally, $\hat{\lambda}$ maximizes the log-likelihood function

$$
l(\lambda)=\sum_{i=1}^{I} \log \operatorname{Pr}_{\lambda}\left(Y_{i}=y_{i}\right)
$$

where

$$
\operatorname{Pr}_{\lambda}\left(Y_{i}=y_{i}\right)=\left(\begin{array}{c}
n_{i} \\
y_{i}
\end{array}\right) p\left(x_{i}\right)^{y_{i}}\left\{1-p\left(x_{i}\right)\right\}^{n_{i}-y_{i}}
$$

is the probability that a binomial variable with size $n_{i}$ and probability $p\left(x_{i}\right)$ (equation 6) equals $y_{i}$. Maximization of the loglikelihood function is made using a Newton-Raphson algorithm. The maximum likelihood estimate of $f$ is obtained by replacing $\mu$ and $\sigma$ by $\hat{\mu}$ and $\hat{\sigma}$ in equation 4 . The maximum likelihood estimate of $g$ is obtained by replacing $v, \kappa$, and $g_{0}$ by $\hat{v}, \hat{\kappa}$, and $\hat{g}_{0}$ in equation 5 . The probability function $p$ is estimated by replacing $\lambda$ by $\hat{\lambda}$ in equations 3 and 6 .

Likelihood ratio tests (LRTs) $(7,35)$ were performed to see (i) whether the directional density function is uniform $(\sigma=0)$ or not $(\sigma>0)$ and (ii) whether the mean distance function is constant over $\left[0^{\circ}, 360^{\circ}[(\kappa=0)\right.$ or not $(\kappa>0)$. For each test, the LRT statistic was parametrically bootstrapped 200 times to get its distribution under the null hypothesis $\sigma=0$ or $\kappa=0$ (27). The $P$ value of the test is given by the proportion of bootstrap LRT statistics greater than the observed LRT statistic.

The $95 \%$ confidence intervals for parameters $s_{0}, \mu, \sigma, \nu, \kappa$, and $g_{0}$ were computed using parametric bootstrap (11). For each site, 200 independent bootstrap datasets were simulated under the fitted parametric model. For each bootstrap data set, the maximum likelihood estimators of the parameters were computed. For any parameter, 200 bootstrap estimates were obtained; the endpoints of the $95 \%$ confidence interval are the 5 th and the 195 th ordered values of the 200 bootstrap estimates.

Goodness-of-fit statistics were computed to see whether the observed number of leaves for Le Neubourg and ThivervalGrignon were suitable values under the fitted parametric models. For each site, 1,000 samples were simulated from the correspond- ing fitted model. For each trap plot, 1,000 simulated numbers of diseased leaves were obtained. Two goodness-of-fit statistics were defined: first, the percentage of trap plots for which the observed numbers of diseased leaves were between the 25th and the 975th ordered values of the simulated numbers of diseased leaves; and second, the percentage of trap plots for which the observed numbers of diseased leaves were between the minimum and the maximum simulated number of diseased leaves. High percentages reflect good fits.

Computations and graphics were made using the R Statistical Software.

\section{RESULTS}

Field experiment. In Le Neubourg, diseased leaves were found in 17 of the 72 trap plots $(23.6 \%)$. The number of diseased leaves per plot ranged from 1 to 85 ; seven plots had only 1 diseased leaf, three plots had 2 to 5 diseased leaves, six plots had 12 to 35 diseased leaves, and one plot had 85 diseased leaves. Spores collected in a given trap plot belonged to a single pathotype, regardless of the number of diseased leaves per plot. The inoculated pathotype 106E139 was identified in 13 of the 17 infected trap plots $(76.5 \%)$. Two of the four naturally contaminated plots were infected by pathotype 233E169-V17, which formed two large foci with 18 and 35 diseased leaves, respectively. The two other naturally contaminated trap plots were infected by pathotype 237E141V17 (one diseased leaf) and 233E137-V17 (three diseased leaves). The maximal dispersal distance of pathotype 106E139 was $175 \mathrm{~m}$ from the source plot. The spatial pattern of the numbers of diseased leaves for Le Neubourg is shown in Figure 2.

In Thiverval-Grignon, diseased leaves were found in 56 of the 187 trap plots $(29.9 \%)$. The number of diseased leaves per trap plot ranged from 1 to $>200 ; 20$ plots had only 1 diseased leaf, 30 plots had 2 to 10 diseased leaves, 5 plots had 14 to 37 diseased leaves, and 1 plot had >200 diseased leaves. Spores collected in a given trap plot belonged to a single physiologic race, regardless of the number of diseased leaves per plot. The inoculated pathotype $106 \mathrm{E} 139$ was identified in 43 of the 56 infected trap plots $(76.8 \%)$. Of the 13 naturally contaminated plots, 8 were infected by pathotype 233E137, 4 by pathotype 237E141-V17, and 1 by pathotype 45E140. Pathotype 237E141-V17 formed one large disease focus (10 infected leaves) whereas the other naturally contaminated trap plots had only 1 to 3 diseased leaves. The maximal dispersal distance of pathotype 106E139 was $225 \mathrm{~m}$ from the source plot. The spatial pattern of the numbers of diseased leaves for ThivervalGrignon is shown in Figure 1.

Nonparametric estimation. The nonparametric estimates of the directional density functions $f$ for Le Neubourg and ThivervalGrignon are shown in Figure $4 \mathrm{~A}$ and $\mathrm{C}$. The nonparametric estimates of the mean distance functions $g^{<}$for Le Neubourg and Thiverval-Grignon are shown in Figure 5A and $\mathrm{C}$. The shapes of these functions suggest that the directional density functions and the mean distance functions are more or less unimodal. Thus, the von Mises form proposed for the anisotropy functions in the parametric model seems to be appropriate.

Parametric estimation. The parametric estimates of the directional density functions $f$ for Le Neubourg and Thiverval-Grignon are shown in Figure 4B and D. The parametric estimates are smoother than the nonparametric estimates (Fig. 4A and C) but show more or less the same main dispersal direction (i.e., spores are mainly dispersed to the bottom to bottom left direction for both experiments). Note that the uniformity of the directional density function $(\sigma=0)$ is rejected for Le Neubourg as well as for Thiverval-Grignon (the bootstrap $P$ value of the LRT is 0 for each experiment). To illustrate the departure of the directional density function from the uniform density, the estimated percentages of spores deposited over the two sectors drawn in Figure $4 \mathrm{D}$ were computed. The estimated percentage for the bottom- 
orientated sector is $18 \%$ instead of $5 \%$. The estimated percentage for the top-orientated sector is $0.1 \%$ instead of $5 \%$.

The parametric estimates of the mean distance functions $g=$ (dashed lines) and $g^{<}$(solid lines) for Le Neubourg and Thiverval-Grignon are shown in Figures $5 \mathrm{~B}$ and $\mathrm{D}$. The parametric estimates of $g^{<}$takes larger values than the nonparametric estimates of $g^{<}$(Fig. 5A and C). However, for both experiments, the directions at which the mean dispersal distance is the largest are more or less the same. Note that the constancy of the mean distance function $(\kappa=0)$ is rejected for Le Neubourg as well as for Thiverval-Grignon (the bootstrap $P$ value of the LRT is 0.04 for Le Neubourg and 0 for Thiverval-Grignon).

Finally, for Le Neubourg and Thiverval-Grignon, the directional density functions on the one hand and the mean distance functions on the other hand do not coincide; that is, directions preferred by spores (Fig. 4) do not correspond to directions at which the mean dispersal distance is the largest (Fig. 5). The difference is significant because the $95 \%$ confidence intervals for parameters $\mu$ and $\nu$ do not overlap (Table 2 shows estimates and confidence intervals of the parameters).

Estimation of the probabilities for leaves to be infected. Maps of probabilities for leaves to be infected (equation 6) obtained by estimating the parametric model are shown in Figures 6 and 7. This sort of map, by combining the directional density function and the mean distance function estimated under the parametric model, allows assessment of the resulting anisotropy.

Goodness-of-fit. A part of the estimated probabilities for leaves to be infected (gray scale) and the observed proportions of infected leaves is shown in Figures 8 and 9 for Le Neubourg and Thiverval-Grignon, respectively. Estimated probabilities and
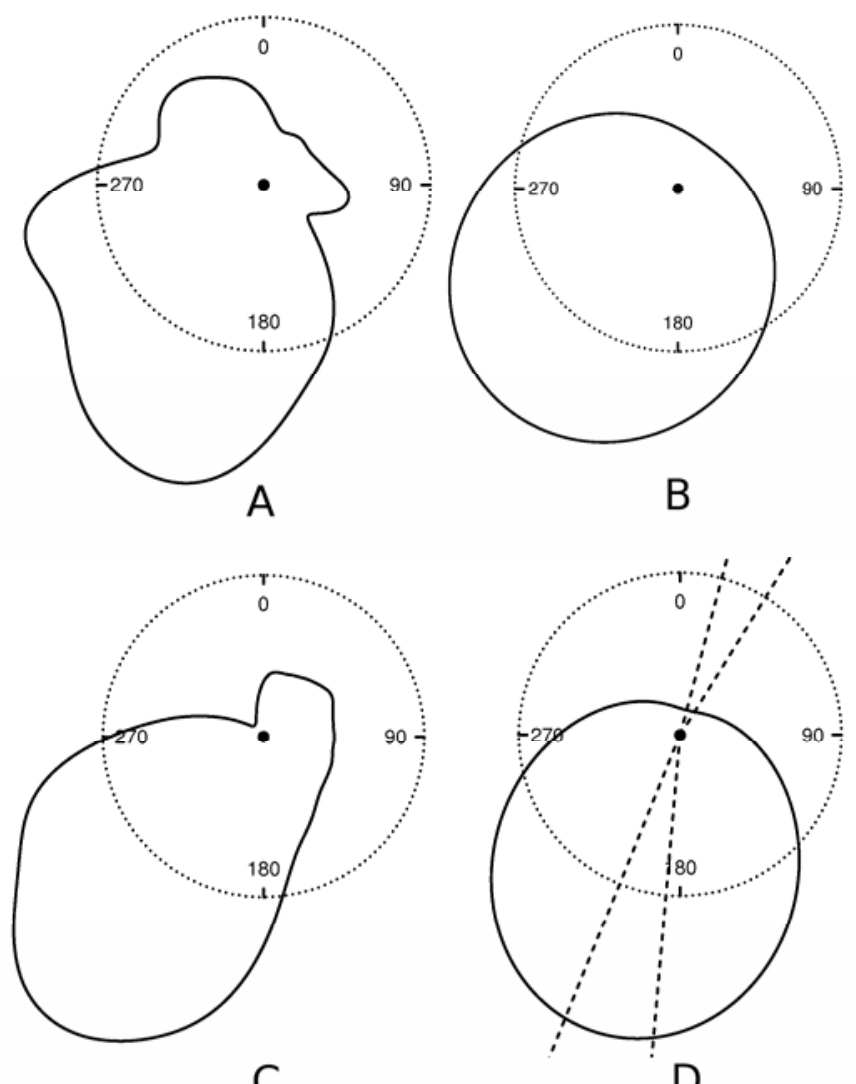

C

Fig. 4. Estimates of the directional density functions (solid lines) for Le Neubourg (top) and Thiverval-Grignon (bottom). Left: estimates obtained under the nonparametric framework; right: estimates obtained under the parametric framework. Point: source location. The directional density function reflects how directions are taken by spores. The estimates can be compared with the uniform directional density function (dotted lines).

observed proportions seem consistent. This is corroborated by goodness-of-fit statistics. The percentages of trap plots with observed numbers of diseased leaves between the 25 th and the 975th ordered values of the 1,000 simulated numbers of diseased leaves are $89 \%$ for Le Neubourg and $90 \%$ for Thiverval-Grignon. The percentages of trap plots with observed numbers of diseased leaves between the minimum and the maximum simulated number of diseased leaves are $97 \%$ for Le Neubourg and $94 \%$ for Thiverval-Grignon. Therefore, the observed numbers of diseased leaves are suitable values under the fitted parametric models. Note that the percentages provided above underestimate the goodnessof-fit because these statistics do not take into account the uncertainty of parameter estimates.

\section{DISCUSSION}

Dispersal experiments in large field plots. The experimental design made of an array of small susceptible wheat plots scattered

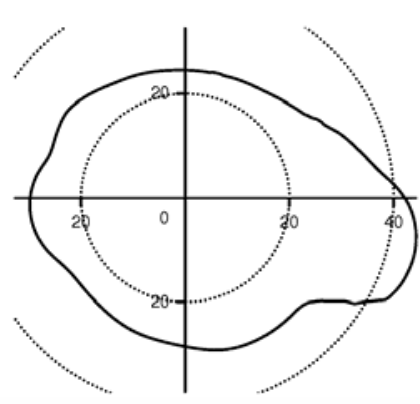

A

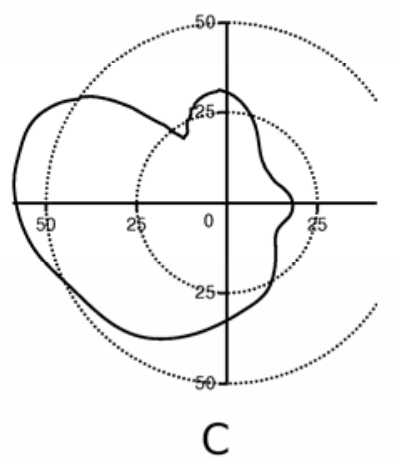

Fig. 5. Estimates of the mean distance functions for Le Neubourg (top) and Thiverval-Grignon (bottom): solid lines for the version $g^{<}$of the mean distance function and dashed lines for the version $g^{=}$which can be computed only in the parametric approach. Left: estimates obtained under the nonparametric framework; right: estimates obtained under the parametric framework. Origin: source location; unit: meter. The mean distance function reflects how far a spore travels in average depending on the direction.

TABLE 2. Maximum likelihood estimates and $95 \%$ confidence intervals (CIs) of the source strength $s_{0}$, the direction parameters $\mu$ and $\sigma$, and the distance parameters $v, \kappa$, and $g_{0}$ included in the parametric model describing the anisotropic dispersal of the yellow rust of wheat in Le Neubourg and Thiverval-Grignon

\begin{tabular}{lccccc}
\hline & \multicolumn{2}{c}{ Le Neubourg } & & \multicolumn{2}{c}{ Thiverval-Grignon } \\
\cline { 2 - 3 } \cline { 5 - 6 } Parameters & Estimate & CI & & Estimate & CI \\
\hline$s_{0}$ & 168 & $(143 ; 208)$ & & 1,371 & $(840 ; 1377)$ \\
$\mu$ & 216 & $(201 ; 227)$ & & 194 & $(184 ; 200)$ \\
$\sigma$ & 1.69 & $(1.28 ; 2.09)$ & & 2.49 & $(2.41 ; 3.37)$ \\
$\nu$ & 89 & $(58 ; 131)$ & & 311 & $(296 ; 321)$ \\
$\kappa$ & 0.69 & $(0.41 ; 1.14)$ & & 1.73 & $(1.51 ; 1.98)$ \\
$g_{0}$ & 147 & $(99 ; 270)$ & & 280 & $(164 ; 375)$ \\
\hline
\end{tabular}


over a large resistant wheat plot allowed us to follow the dispersal of yellow rust from a source plot during a single generation of the pathogen. The discontinuous pattern of the trapping area decreased the number of data (infected leaves) compared with the data which would have been obtained with a continuous sampling scheme. However, discontinuous sampling on a regular grid allowed us to observe disease all over the experimental area without any strong presupposition on the main distance of dispersal.

Mapping disease spread up to a distance of 125 and $225 \mathrm{~m}$ from the source in Le Neubourg and Thiverval-Grignon, respectively, was possible in spite of a fairly small trapping area. This is far beyond distances over which yellow rust dispersal already has been documented in field experiments $(12,38)$, and also far beyond the distance on which faba bean rust dispersal was studied using a similar, discontinuous design (37). Based on knowledge

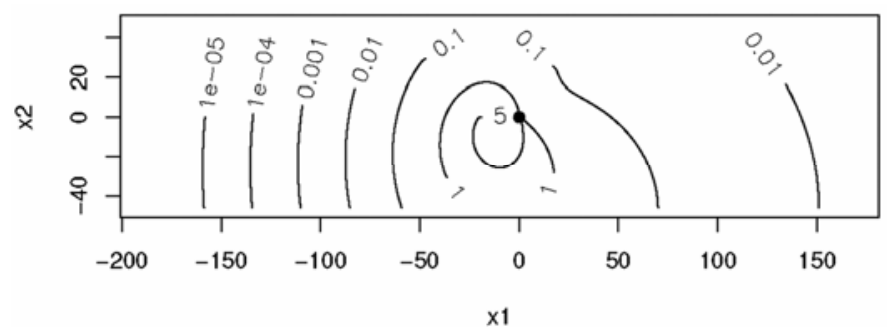

Fig. 6. Map of the risk of infection of leaves by the source plot in Le Neubourg. Figures on the isopaths are the probabilities (\%), estimated under the parametric model, for leaves to be infected by the source plot. Point: source plot location; axes unit: meter.

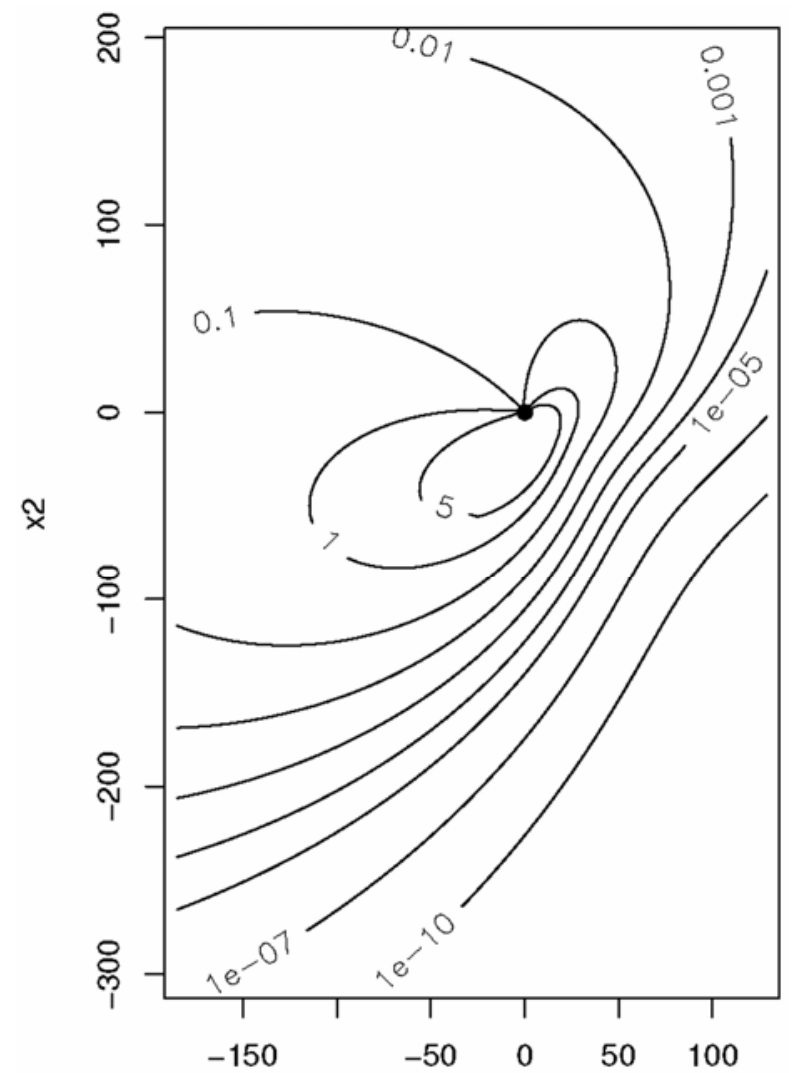

$\mathrm{x} 1$

Fig. 7. Map of the risk of infection of leaves by the source plot in ThivervalGrignon. Figures on the isopaths are the probabilities (\%), estimated under the parametric model, for leaves to be infected by the source plot. Point: source plot location; axes unit: meter. of the genetic structure of the populations of the pathogen, we were able to identify contamination from external sources of inoculum; on both experimental sites, $<25 \%$ of the infected trap plots were contaminated by such sources of inoculum. After a strong epidemic of yellow rust in France in 1999, the severity of yellow rust was low in 2002, especially early in the crop season, according to the national disease survey made by the French Plant Protection Services; moreover, pathotype identification from samples collected in northern and western France confirmed that pathotype 237E141-V17, found in both experimental locations, was dominant in the natural pathogen population $(22, \mathrm{C}$. de Vallavieille-Pope, personal communication). Because the experiment was not designed to quantify external contamination, no attempt was made to interpret the level of this contamination and to trace it back to its origin. Due to the well-established longdistance dispersal ability of rust spores (5), the external spores might come from a neighboring field as well as from a very distant region. Migration of yellow rust spores over long distances (e.g., from England) has been documented in the regions where we did the experiment (3).

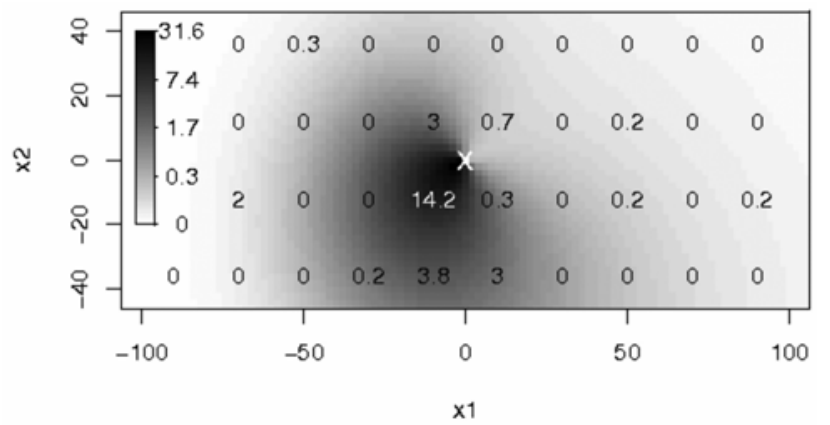

Fig. 8. Part of the map of the estimated probabilities (\%) for leaves to be infected (gray scale) and the observed proportions of infected leaves for Le Neubourg. The probabilities for leaves to be infected were estimated under the parametric model. Cross at the origin: source location; axes unit: meter.

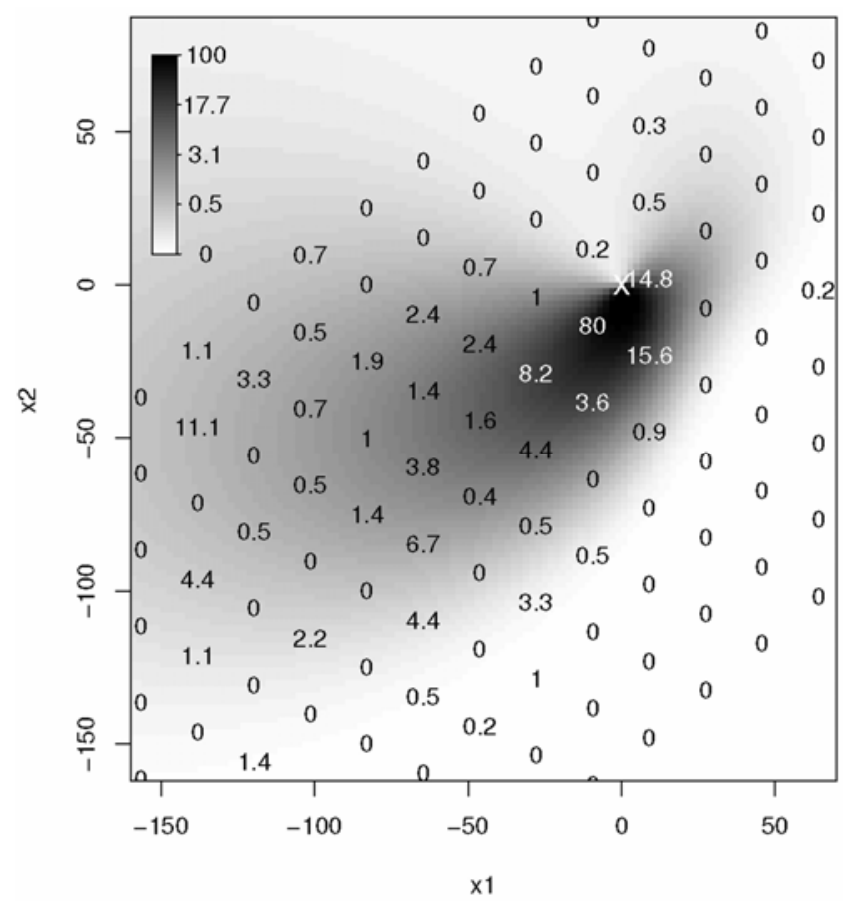

Fig. 9. Part of the map of the estimated probabilities (\%) for leaves to be infected (gray scale) and the observed proportions of infected leaves for Thiverval-Grignon. The probabilities for leaves to be infected were estimated under the parametric model. Cross at the origin: source location; axes unit: meter. 
Anisotropy of spore dispersal. The anisotropy of disease spread was evidenced on maps showing the results of the two experiments (Figs. 1 and 2). The differences in the pattern of disease spread between the two sites were caused by local differences in weather conditions during the experiment. Disease spread is the result of spore dispersal and leaf infection; therefore, the anisotropy of disease spread could be caused by (i) anisotropy of airborne spore dispersal or (ii) variations of leaf receptivity from trap plot to trap plot. Individual variations in the leaf receptivity were included in a model describing the dispersal of wheat leaf rust on short distance around a diseased leaf (40). In the present, large-scale experiment, we considered that these individual variations were negligible and that the average leaf receptivity in a trap plot was the same all over the experiment area. Accordingly, only cause (i) was integrated in the modeling framework.

The purpose of the modeling approach was not to perform a mechanistic interpretation of the local variations in spore dispersal; rather, its purpose was to propose a generic modeling framework suitable for the analysis of anisotropy of airborne spore dispersal in a variety of experimental situations, varying in terms of weather conditions and sampling schemes. Statistical tests (tests of $\sigma=0$ and $\kappa=0$ ) confirmed the significance of the anisotropy that was visually evidenced on maps showing the results of the experiments (Figs. 1 and 2).

Anisotropy in density and in distance. The anisotropy of spore dispersal in density and in distance is not clearly visible on mapped results of the experiments; however, it was shown and quantified by the statistical analysis. To characterize the anisotropy of spore dispersal in density and in distance, two anisotropy functions were introduced and estimated: the directional density function and the mean distance function. The statistical analysis performed for the two experiments showed that the anisotropy functions can differ in their orientation.

The anisotropy functions are complementary tools to the widely used gradient curves $(15,17,24,38)$ to quantify dispersal in a field experiment. The gradient curve allows the measurement of the decrease in disease intensity with distance from the spore source, whereas the anisotropy functions allow for assessment of how dispersal directions are preferred by spores and how far, on average, a spore travels in the various directions.

The anisotropy functions are defined without a priori specification of a model. However, for estimating them, two models were proposed: a nonparametric one and a parametric one. Data from the field experiments presented in this article did not provide accurate nonparametric estimators of the anisotropy functions because, ideally, data should be deposition locations for a set of randomly sampled spores. For that reason, the nonparametric approach was used principally to determine the main directions and the shapes of the anisotropy functions. This information then was used to specify the form of the anisotropy functions in the parametric model. The unimodal von Mises function was used; however, more flexible functions such as mixtures of von Mises functions and elliptical functions could be used. However, such functions require more parameters, and problems of identifiability may occur. Increasing the quantity of data (that is, increasing the quantity of trap plots) should allow estimation of more flexible parametric anisotropy functions and allow more accurate estimation. Note that, contrary to the nonparametric approach, the parametric one is adapted to available data because the parametric model describes the behavior of the measured variable (i.e., the number of diseased leaves per trap plot).

It is important to note that the estimates of the anisotropy functions are conditional on the spatial distribution of the sampling points. In other words, the estimates of the anisotropy functions are influenced by the spatial structure of the sampling scheme. In the nonparametric approach, the estimated anisotropy functions are given for spores deposited in the sampling area and, ideally, the sampling area should be circular to avoid biases of estimation depending on the direction. In the parametric approach, the need for a circular sampling area is less important because the parametric model provides an extrapolation for points outside the sampling area. Indeed, the parametric model is fitted such that the estimated probabilities of infection are coherent with the observed proportion of infected leaves at the sampling points; for points outside the sampling area, the probabilities of infection are extrapolated according to the parametric model. Therefore, if the parametric model is true, the shape of the sampling area could influence the accuracy of the estimates but should not introduce bias of estimation. Any concern about the influence of the shape of the sampling area on the estimation is linked with the following question: how confident are we in the parametric model? The goodness-of-fit of the model suggests that we can be reasonably confident. Nevertheless, one must be conscious that, even if the parametric model seems suitable, it certainly is not flexible enough to describe the true behavior of the dispersal at shorter and larger distances from the source than the sampled ones; and, even if it was flexible enough, we do not have data at such distances. So, in practice, if the trap plots had been distributed at shorter distances from the source, for example, we might have obtained different estimates of the anisotropy functions. The argument developed in this paragraph has an analogue: the half distance $(15,24)$, which generally is provided when a gradient curve is fitted, depends on the range of sampled distances. To conclude, the inference on the anisotropy functions must be considered as reliable only within the limits of the experimental scale. Moreover, in order to avoid the aforementioned problems of inference when applying the nonparametric and parametric approaches proposed in this article, future experiments intended to investigate anisotropic spread of disease should be done using circular sampling schemes with a dense network of trap plots (100 to 150 trap plots seems to be enough for estimating the anisotropic functions, but the estimation accuracy increases with the number of trap plots).

Estimating the two anisotropy functions allowed us to quantify and differentiate the anisotropy in density and the anisotropy in distance. To go further in the understanding of the anisotropy of the disease spread, a study exploring the link between the estimated anisotropy functions and relevant factors could be carried out. Factors of interest could be, for instance, topography, wind speed, wind direction, and the interactions between the temporal sequence of the wind and the temporal sequence of microclimatic factors, because temperature, leaf wetness, light intensity, and rain events can affect spore production (25), spore release (36), and infection efficiency (9) for yellow rust. Thus, the dissociation of the two anisotropies should help to understand the correlations between spread patterns and microclimatic variables.

\section{ACKNOWLEDGMENTS}

This project was supported in part by CTPS (grant no. B04450) and INRA (EpiEmerge program). We thank G. Lelarge (LPA "Gilbert Martin," Le Neubourg), B. de Franssus (Experimental Farm, INA-PG, ThivervalGrignon), and J. Troizier (INRA, Thiverval-Grignon) for the management of the experimental plots; E. Klein for his contribution to the design of the experiment; M. Leconte for guidance with pathotype identification; L. Gérard for technical assistance; J. Chadœuf for his comments on the modelling; and senior editor N. McRoberts and two anonymous reviewers for their comments and suggestions on the article.

\section{LITERATURE CITED}

1. Aylor, D. E. 1990. The role of intermittent wind in the dispersal of fungal pathogens. Annu. Rev. Phytopathol. 28:73-92.

2. Aylor, D. E. 1998. The aerobiology of apple scab. Plant Dis. 82:838849 .

3. Bayles, R. A., Flath, K., Hovmøller, M. S., and De Vallavieille-Pope, C. 2000. Breakdown of the Yr17 resistance to yellow rust of wheat in northern Europe. Agronomie 20:805-811. 
4. Bosq, D., and Lecoutre, J.-P. 1987. Théorie de l'Estimation Fonctionnelle. Economica, Paris.

5. Brown, J. K. M., and Hovmøller, M. S. 2002. Aerial dispersal of pathogens on the global and continental scales and its impact on plant disease. Science 297:537-541.

6. Cammack, R. H. 1958. Factors affecting infection gradients from a point source of Puccinia polysora in a plot of Zea mays. Ann. Appl. Biol. 46:186-197.

7. Dacunha-Castelle, D., and Duflo, M. 1982. Probabilités et Statistiques; Problèmes à Temps Fixe. Masson, Paris.

8. Danial, D. L., Broers, L. H. M., and Parlevliet, J. E. 1993. Does interplot interference affect the screening of wheat for yellow rust resistance? Euphytica 70:217-224.

9. De Vallavieille-Pope, C., Huber, L., Leconte, M., and Bethenod, O. 2002. Preinoculation effect of light quantity on infection efficiency of Puccinia striiformis and P. triticina on wheat seedlings. Phytopathology 92:13081314.

10. De Vallavieille-Pope, C., Rouzet, J., Leconte, M., Delos, M., and Mistou, M. N. 2000. La rouille jaune du blé en France: Des épidémies déclenchées par une nouvelle race, un hiver doux et un printemps humide. Phytoma 527:22-29.

11. Efron, B., and Tibshirani, R. J. 1993. An Introduction to the Bootstrap. Chapman and Hall, New York.

12. Emge, R. G., and Shrum, R. D. 1976. Epiphytology of Puccinia striiformis at five selected locations in Oregon during 1968 and 1969. Phytopathology 66:1406-1412.

13. Enjalbert, J., Duan, X., Leconte, M., Hovmøller, M. S., and De Vallavieille-Pope, C. 2005. Genetic evidence of local adaptation of wheat yellow rust (Puccinia striiformis f. sp. tritici) within France. Mol. Ecol. 14:2065-2073.

14. Fisher, N. I. 1995. Statistical Analysis of Circular Data. Cambridge University Press.

15. Fitt, B. D. L., Gregory, P. H., Todd, A. D., McCartney, H. A., and MacDonald, O. C. 1987. Spore dispersal and plant disease gradients; A comparison between two empirical models. J. Phytopathol. 118:227-242.

16. Gregory, P. H. 1945. The dispersion of air-borne spores. Trans. Brit. Mycol. Soc. 28:26-72.

17. Gregory, P. H. 1968. Interpreting plant disease dispersal gradients. Annu. Rev. Phytopathol. 6:189-212.

18. Hastie, T. J., and Tibshirani, R. J. 1990. Generalized Additive Models. Chapman and Hall, London.

19. Johnson, R., Stubbs, R. W., Fuchs, E., and Chamberlain, N. H. 1972. Nomenclature for physiologic races of Puccinia striiformis infecting wheat. Trans. Brit. Mycol. Soc. 58:475-480.

20. Kingsolver, C. H., Peet, C. E., and Underwood, J. F. 1984. Measurement of the epidemiologic potential of wheat stem rust: St. Croix, U.S. Virgin Islands, 1954-57. Pa. Agric. Exp. Stn Bull. 854:1-21.

21. Klein, E. K., Lavigne, C., Foueillassar, X., Gouyon, P. H., and Larédo, C. 2003. Corn pollen dispersal: Quasi-mechanistics models and field experiments. Ecol. Monogr. 73:131-150.

22. Le Henaff, G., Oste, B., Wilhem, E., Faure, A., Moinard, J., Lepoutre, P., and Pillon, O. 2003. Bilan phytosanitaire 2001-2002 des céréales. Phytoma Def. Veg. 556:22-26.

23. Loader, C. R. 1999. Bandwidth selection: Classical or plug-in? Ann. Stat. 27:415-438.
24. McCartney, H. A., and Fitt, B. D. L. 1985. Construction of dispersal models. Adv. Plant Pathol. 3:107-143.

25. McGregor, A. J., and Manners, J. G. 1985. The effect of temperature and light intensity on growth and sporulation of Puccinia striiformis on wheat. Plant Pathol. 34:263-271.

26. McIntosh, R. A., Wellings, C. R., and Park, R. F. 1995. Wheat Rusts: An Atlas of Resistance Genes. CSIRO, Melbourne, Australia.

27. McLachlan, G. J. 1987. On bootstrapping the likelihood ratio test statistic for the number of components in a normal mixture. J. R. Stat. Soc. C 36:318-324

28. McNeal, E. H., Konzak, C. F., Smith, E. P., Tate, W. S., and Russel, T. S. 1971. A uniform system for recording and processing cereal research data. Pages 34-121 in: Agric. Res. Serv. Bull. U.S. Dep. Agric. Washington, DC.

29. McRoberts, N., Hughes, G., and Madden, L. V. 2003. The theoretica basis and practical application of relationships between disease intensity measurements in plants. Ann. Appl. Bio. 142:191-211.

30. Oerke, E. C., Dehne, H. W., Schönbeck, F., and Weber, A. 1994. Crop Production and Crop Protection. Estimated Losses in Major Food and Cash Crops. Elsevier, Amsterdam.

31. Rapilly, F. 1977. Réflexions sur les notions de propagule et d'unité de dissémination en épidémiologie végétale: Cas des champignons parasites des plantes cultivées. Ann. Phytopathol. 9:161-176.

32. Rapilly, F. 1979. Yellow rust epidemiology. Annu. Rev. Phytopathol. 17:59-73.

33. Rapilly, F., Fournet, J., and Skajennikoff, M., 1970. Etudes sur l'épidémiologie et la biologie de la rouille jaune du blé Puccinia striiformis Westend. Ann. Phytopathol. 2:5-31.

34. Roelfs, A. P, Singh, R. P., and Saari, E. E. 1992. Rust Diseases of Wheat: Concepts and Methods of Disease Management. CIMMYT, Mexico.

35. Rohatgi, V. K. 2003. Statistical Inference. Dover Publication, Mineola.

36. Sache, I. 2000. Short-distance dispersal of wheat rust spores by rain and wind. Agronomie 20:757-767.

37. Sache, I., and Zadoks, J. C. 1995. Spread of faba bean rust over a discontinuous field. Eur. J. Plant Pathol. 102:51-60.

38. Sackett, K. E., and Mundt. C. C. 2005. Primary disease gradients of wheat stripe rust in large field plots. Phytopathology 95:983-991.

39. Silverman, B. W. 1986. Density Estimation for Statistics and Data Analysis. Chapman and Hall, London.

40. Soubeyrand, S., Sache, I., Lannou, C., and Chadœuf, J. 2007. A frailty model to assess plant disease spread from individual count data. J. Data Sci. 5:67-83

41. Stockmarr, A. 2002. The distribution of particles in the plane dispersed by a simple 3-dimensional diffusion process. J. Math. Biol. 45:461-469.

42. Tufto, J., Engen, S., and Hindar, K. 1997. Stochastic dispersal processes in plant populations. Theor. Pop. Biol. 52:16-26.

43. Underwood, J. F., Kingsolver, C. H., Peet, C. E., and Bromfield, K. R. 1959. Epidemiology of stem rust of wheat: III. Measurements of increase and spread. Plant Dis. Rep. 43:1154-1159.

44. Villaréal, L. M. M. F., Lannou, C., De Vallavieille-Pope, C., and Neema, C. 2002. Genetic variability in Puccinia striiformis f. sp. tritici populations sampled on a local scale during natural epidemics. Appl. Environ. Microbiol. 68:6138-6145.

45. Zadoks, J. C., and Van den Bosch, F. 1994. On the spread of plant disease: A theory on foci. Annu. Rev. Phytopathol. 32:503-521. 